\title{
Sleep and Olfaction among Older Adults
}

\author{
V. Eloesa McSorley ${ }^{\mathrm{a}}$ Jayant Pinto $^{\mathrm{b}} \quad$ L. Philip Schumm $^{\mathrm{a}}$ Kristen Wroblewski $^{\mathrm{a}}$ \\ David Kern $^{d}$ Martha McClintock ${ }^{c}$ Diane S. Lauderdale ${ }^{a}$ \\ ${ }^{a}$ Department of Public Health Sciences, ${ }^{b}$ Pritzker School of Medicine, and ' Department of Psychology, University of \\ Chicago, and ${ }^{\mathrm{d}}$ Northeastern Illinois University, College of Arts and Sciences, Chicago, IL, USA
}

\section{Keywords}

Olfaction · Sleep · Actigraphy · Aging

\begin{abstract}
Background: Sleep and olfaction are both critical physiological processes that tend to worsen with age. Decline in olfaction can be an early indicator of neurodegenerative diseases, whereas poor sleep quality is associated with reduced physical and mental health. Given associations with aging-related health declines, we explored whether variations in sleep were associated with olfactory function among older adults. Methods: We assessed the relationship between sleep characteristics and olfaction among 354 community-dwelling older adults. Olfaction was measured using a validated field and survey research tool. Sleep characteristics were measured using wrist actigraphy and with self-report of sleep problems. We fit structural equation models of latent constructs of olfaction based on olfactory task items and let this be a function of each sleep characteristic. Results: Actigraph sleep quality measures were associated with odor identification, but not with odor sensitivity. Longer duration sleepers had worse odor sensitivity compared to medium (58 h) sleepers, but sleep duration was not associated with odor identification. Reported sleep problems and reported usual duration were not associated with olfaction. Conclusions: Diminished sleep quality was associated with reduced ca-
\end{abstract}

\section{KARGER}

๑) 2017 S. Karger AG, Basel

E-Mail karger@karger.com

www.karger.com/ned pacity to identify odors. Determining whether this is a causal association will require further study and longitudinal data.

(c) 2017 S. Karger AG, Basel

\section{Introduction}

Olfaction is critical for human health and is involved in psychosocial functioning, nutrition, social ties, memory, emotion, mood, and overall well-being [1-3]. Olfactory dysfunction can be harmful through decreased nutritional status, worse emotional and physical well-being, increased depressive symptoms, and social isolation [4-8].

Olfaction has been shown to progressively decline after age $57[9,10]$, with up to $80 \%$ of those over age 80 showing impairment [11]. Olfactory dysfunction is an early indicator of neurodegenerative diseases including Alzheimer's, Parkinson's, and Huntington's disease [11] and also predicts mortality among cognitively intact older adults [12-15]. Olfactory impairment is also more prevalent among men, smokers, African-Americans, Hispanics, those with lower SES and lower cognition scores, and stroke victims $[13,16,17]$. As olfaction is an indicator of neural degeneration, aging, and death, determining the underlying mechanisms of these relationships is important to understanding health trajectories.

Dr. Diane S. Lauderdale

Department of Public Health Sciences, University of Chicago 5841 S Maryland Ave

MC 2000, Room W250, Chicago, IL 60637 (USA)

E-Mail lauderdale@ health.bsd.uchicago.edu 
Like olfaction, sleep is a physiological process crucial to brain health. Older adults experience increased sleep disturbances including more wake after sleep onset (WASO), greater sleep fragmentation, and poorer selfreported sleep quality including insomnia symptoms [18, 19]. Deep sleep and REM sleep also decline with age [20]. Though findings are inconsistent about sleep duration and mortality (Kurina et al. [21]), sleep disturbances have consistently been associated with chronic disease, overall physical and mental health, cognitive function, and mortality [18, 22-26].

Given the relationships of olfactory decline and sleep with aging-related health problems, we sought to explore whether variations in sleep were associated with olfactory dysfunction among older adults.

Previous studies have investigated the relationship between sleep and olfaction in humans, including olfactory function and memory consolidation of odors during sleep [27-30] and memory consolidation during sleep using olfactory cues [31-34]. However less attention has been given to whether variations in sleep are associated with olfactory function more generally. Two experimental studies found that sleep deprivation was associated with a worse ability to identify odors among adults [27, 35], consistent with neuroimaging studies showing that sleep deprivation leads to decreases in the cerebral metabolic rate for glucose in the prefrontal cortex, including the orbitofrontal region, which is highly involved in olfactory processing [36]. However, the relationship between sleep and olfactory function has not been assessed in a population setting among older adults, the group that experiences increased prevalence of olfactory dysfunction and disordered sleep.

The current analysis examines the relationship between actigraph and self-reported measures of sleep duration and quality with objectively assessed olfactory function among a subsample of a national study of older adults.

\section{Methods}

\section{Study Sample}

The National Social Life, Health, and Aging Project (NSHAP) is a nationally representative sample of community-dwelling older adults born between 1920 and 1947 and their consenting spouses, regardless of their age. Survey data and biomeasures were collected via in-person at-home interviews.

In wave $2(2010 / 2011)$, the NSHAP population was divided into 6 subgroups to allocate participants to additional modules [37]. Four subgroups $(n=2,304)$ were selected to receive the Olfactory Function Field Exam (OFFE), which was developed to mea- sure the olfactory function in survey research [38]. Two subgroups $(n=1,117)$ were selected to participate in the sleep module. A total of 572 individuals were selected to receive both the OFFE and the sleep module. There were 354 participants aged $62-90$ who consented to both modules and provided complete data (Fig. 1).

\section{Measures}

Olfaction: the OFFE includes measures of odor identification and sensitivity. Odor identification was assessed using a field survey version of a validated test [39]. Participants were presented with 5 odor-filled felt tip pens and were instructed to identify each odor from a selection of 4 word/picture options. The number of correctly identified odors yielded a score from 0 to 5 .

The odor sensitivity module of the OFFE assessed participants' capacities to detect $n$-butanol, a common testing odorant. Participants were presented with 3 felt tip pens and asked to select the one pen that contained $n$-butanol. Six sets with increasing concentrations of $n$-butanol in one pen were presented. Scores of 0-6 represent how many concentrations were correctly detected. This screening test is reliable and highly correlated $(r=0.92)$ with psychophysical olfactory sensitivity testing among older adults [40]. A detailed protocol of olfactory data collection, including interviewer training, has been described elsewhere [41].

Sleep: sleep characteristics were collected via self-report and wrist actigraphy. Actigraphs (Actiwatch Spectrum model, Phillips Respironics) were worn by study participants for a 72-h period. Participants were asked to push an event marker each night when they started trying to sleep and when they awoke. The event markers, activity counts, and ambient light data (recorded by the actigraph) were used to manually determine the main rest interval for each 24-h period. Actiwatch software calculated sleep metrics from the pattern of activity counts within each rest interval. We used metrics frequently derived from actigraphy: total sleep duration - the sum of 15-s epochs scored as sleep during the main sleep interval; WASO - total minutes awake during the main sleep interval; and fragmentation - an index of restlessness expressed as a percentage. Averages of each were calculated from the 3 nights. A detailed protocol of sleep data collection, including quality control measures, has been described elsewhere [42].

Self-reported sleep duration was assessed via a question asking how many hours participants slept each night. Sleep problems similar to insomnia symptoms (i.e., frequency of trouble falling asleep, trouble waking, waking too early, and restorative sleep) were combined to create a troubled sleep scale. The scale ranges from 0 to 8 , with higher scores indicating more insomnia symptoms, as described in detail elsewhere [43].

Cognitive function: cognitive function was measured using the Montreal Cognitive Assessment (MoCA) [44] adapted for survey administration (MoCA-SA) $[45,46]$. The MoCA was developed to assess mild cognitive impairment (MCI) across key cognitive domains and was shown to have a $90 \%$ sensitivity in detecting clinically diagnosed MCI [44]. The instrument is more sensitive to variation in cognitive function than screeners designed to identify severe impairment, such as the Mini-Mental State Exam. The MoCA-SA is highly correlated $(r=0.973)$ with the full MoCA [45, 46].

Additional covariates: demographic and control variables included age, gender, body mass index, race/ethnicity (white, Hispanic, black, other), current smoking, depressive symptoms, med- 
Fig. 1. Flow diagram for participants in the sleep-olfaction substudy of the National Social Life, Health, and Aging Project (NSHAP).
3,377 NSHAP wave 2 participants

545 only selected for sleep study 1,732 only selected for Olfactory function Field exam (OFFE)

572 randomly selected for OFFE and sleep study

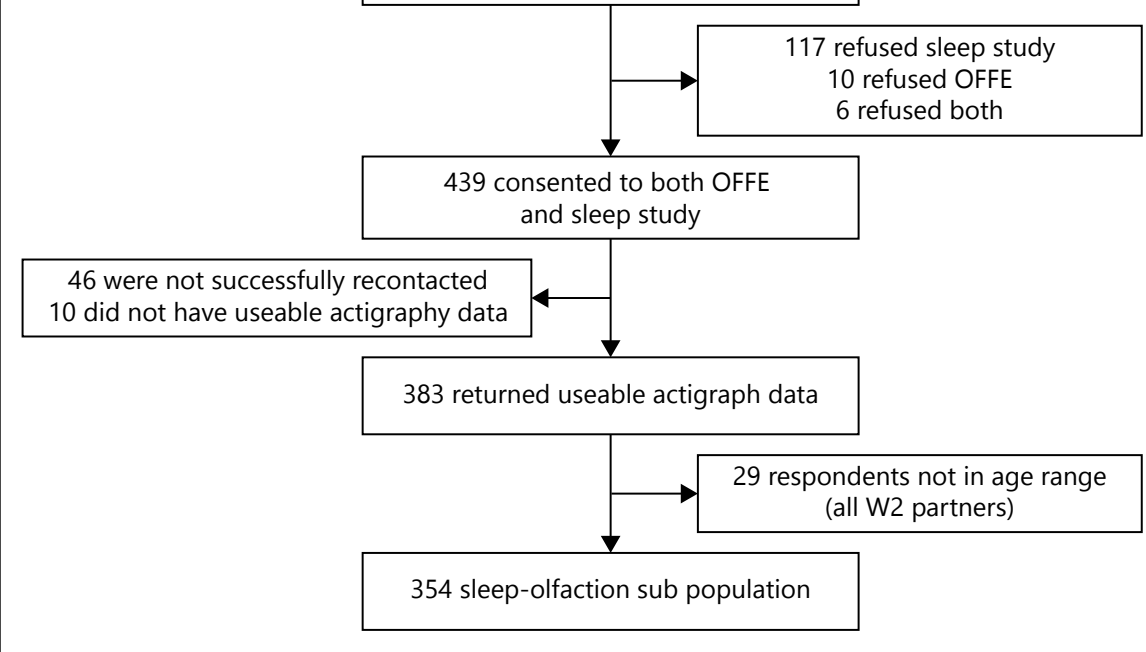

ications, and a comorbidity index. Body mass index was calculated from direct measures $\left(\mathrm{kg} / \mathrm{m}^{2}\right)$. Depressive symptoms were assessed using the Center for Epidemiologic Studies Depression scale, which assesses the frequency of depressive feelings over the past week. Medications were physically brought to the interviewers and recorded. We include indicators for the current usage of antidepressants and hypnotics. Comorbidities were summarized using a modified version of the Charlson Comorbidity Index [47], as implemented in NSHAP [48].

\section{Statistical Analysis}

To assess the relationship between sleep characteristics and odor identification, we fit a generalized structural equation model to the 5 observed olfactory identification items assuming a single latent construct of odor identification through a logistic regression (i.e., a 1-parameter item-response model). This model was then extended by specifying odor identification to be a function of each sleep characteristic separately, first only adjusting for age and gender and then adjusting for additional covariates. Due to evidence in the literature of a U-shaped relationship between sleep time and some health outcomes, we also tested a 3-level categorical variable for sleep time $[21,49]$. The beta coefficient of the main independent variable (the particular sleep characteristic) represents the average change in performance on the odor identification construct for each additional unit change of the sleep characteristic. The same analysis was performed for odor sensitivity.

As a secondary analysis, we assessed potential mediation of the relationship between sleep characteristics and odor identification via cognition. To assess potential mediation, we augmented the structural equation models to allow us to consider possible mediat- ing effects of cognition using the widely implemented product method [50].

All analyses took into account the study design and sampling weights to account for probabilities of selection and nonresponse [51]. All data were analyzed using Stata version 13.0 (StataCorp LP, College Station, TX, USA).

\section{Results}

Table 1 summarizes the demographic, health, and sleep characteristics of the olfaction-sleep subsample. Actigraph total sleep time averaged $7.2 \mathrm{~h}$, WASO averaged $37.5 \mathrm{~min}$, and the fragmentation index averaged $14.2 \%$. Average self-reported sleep was $7.4 \mathrm{~h}$ and the average troubled sleep scale was 2.7. Just over half were able to identify all 5 odors (51.4\%); however, only $7.1 \%$ were able to detect the weakest concentration of $n$-butanol. The distribution of covariates in the olfaction-sleep subsample was generally similar to the larger study population (online suppl. Table S1; see www.karger.com/ doi/10.1159/000479066).

Table 2 presents the coefficients for the sleep characteristics in the age- and gender-adjusted (model 1) and fully adjusted (model 2) structural equation models for both 
Table 1. Demographics of the NSHAP wave 2 olfaction-sleep subsample $(n=354)$

\begin{tabular}{|c|c|c|}
\hline Characteristic & Weighted value & $n$ \\
\hline Age, years, mean (SD) & $71.7(7.5)$ & 354 \\
\hline Female, $\%$ & 53.6 & 354 \\
\hline Race, $\%$ & & 354 \\
\hline White & 82.3 & \\
\hline African-American & 6.2 & \\
\hline Hispanic & 6.3 & \\
\hline Other & 5.2 & \\
\hline Modified charlson comorbidity, \% & & 354 \\
\hline 0 & 48.1 & \\
\hline 1 & 25.4 & \\
\hline 2 & 14.0 & \\
\hline $3+$ & 12.4 & \\
\hline Montreal Cognitive Assessment - & & \\
\hline Survey Adapted, $0-20$ scale, mean (SD) & $14.2(3.5)$ & 354 \\
\hline Body mass index, $\mathrm{kg} / \mathrm{m}^{2}$, mean (SD) & $29.1(5.50)$ & 345 \\
\hline Current smoker, \% & 13.6 & 354 \\
\hline $\mathrm{CES}^{\mathrm{1}}{ }^{1}$, mean $(\mathrm{SD})$ & $7.3(3.3)$ & 354 \\
\hline \multicolumn{3}{|l|}{ Medication usage, $\%$} \\
\hline Antidepressants & 17.4 & 334 \\
\hline Sleep aids & 8.7 & 334 \\
\hline Actigraph-measured sleep & & 354 \\
\hline $\mathrm{WASO}^{2}$, min, mean $(\mathrm{SD})$ & $37.5(22.6)$ & \\
\hline Total sleep time, h, mean (SD) & $7.2(1.4)$ & \\
\hline$<5 \mathrm{~h}, \%$ & 5.5 & \\
\hline $5-8 \mathrm{~h}, \%$ & 70.2 & \\
\hline$>8 \mathrm{~h}, \%$ & 24.3 & \\
\hline Fragmentation, $n(\%)$ & $14.2(5.9)$ & \\
\hline Self-reported sleep duration, h, mean (SD) & $7.4(1.3)$ & 287 \\
\hline Troubled Sleep Scale ${ }^{3}$, mean (SD) & $2.7(2.1)$ & 354 \\
\hline \multicolumn{3}{|l|}{ Olfactory Function Field Exam } \\
\hline Odor identification (score) $)^{4}, \%$ & & 354 \\
\hline 0 & 2.9 & \\
\hline 1 & 1.3 & \\
\hline 2 & 6.0 & \\
\hline 3 & 7.8 & \\
\hline 4 & 30.7 & \\
\hline 5 & 51.3 & \\
\hline Odor sensitivity (score) $)^{5}, \%$ & & 354 \\
\hline 0 & 7.1 & \\
\hline 1 & 8.5 & \\
\hline 2 & 12.4 & \\
\hline 3 & 15.9 & \\
\hline 4 & 23.8 & \\
\hline 5 & 25.3 & \\
\hline 6 & 7.1 & \\
\hline
\end{tabular}
Scale.

${ }^{1}$ CES-D (range 0-22): Center for Epidemiologic Study Depression

${ }^{2}$ Wake after sleep onset.

${ }^{3}$ Troubled Sleep Scale (range $\left.0-8\right)$ is a combined metric $(0=$ never/ rarely, 1 = sometimes, 2 = most of the time) from 4 questions: feeling rested in the morning, trouble falling asleep, trouble waking during the night and trouble waking too early.

${ }^{4}$ Odor identification (range $0-5$ ) is measured via correct identification of five odorants: rose, leather, orange, fish, and peppermint.

${ }^{5}$ Odor sensitivity (range $0-6$ ) is assessed by participants' capacities to detect n-butanol. odor identification and odor sensitivity. The outcome for each is the latent variable construct of the particular odor scale, where a higher score indicates better performance. In the age- and gender-adjusted models, both WASO and fragmentation were significantly inversely associated with odor identification. In the fully adjusted models, each additional minute of WASO was associated with a 0.013 worse odor identification score $(p<0.01)$, and each additional percent of fragmentation was associated with a 0.039 worse odor identification score $(p=0.09)$.

The troubled sleep scale, self-reported sleep duration, or actigraph total sleep time was not associated with odor identification in adjusted models. Modeled as a categorical variable, there was no evidence of a U-shaped relationship between the actigraph-measured total sleep time and odor identification

Also, WASO, fragmentation, self-reported sleep duration, or the troubled sleep scale score was not associated with odor sensitivity. Modeled as a continuous variable, actigraph total sleep time was inversely associated with odor sensitivity; each additional hour of sleep time was associated with a 0.152 worse odor sensitivity score $(p=0.02)$. When modeled as a categorical variable, long sleepers (subjects averaging $8 \mathrm{~h}$ or more per night) were less able to detect odors compared to medium (5-8 h) sleepers, with marginal significance $(\beta=-0.426, p=0.08)$.

We assessed potential mediation by cognition between both WASO and fragmentation with odor identification (Table 3). The indirect association represents the portion of the association between the sleep measure and odor identification that is mediated by cognition. Only small portions of the relationships for WASO and fragmentation with odor identification were mediated by cognition $(-0.002, p=0.14$ and $-0.006, p=0.22$, respectively).

\section{Discussion}

In our assessment of sleep and olfaction in community-dwelling older adults, we found a positive association between 2 actigraph measures of sleep disruption and worse ability to correctly identify odors, after adjusting for demographics and comorbidities. We did not find an association between insomnia symptoms or sleep duration, measured either by self-report or actigraphy, and measured by the ability to identify odors. We also did not find an association between sleep disruption or insomnia symptoms and odor sensitivity. However, we did observe an unexpected inverse association between total sleep time (measured with actigraphy) and odor sensitivity. 
Table 2. Associations between sleep characteristics and odor identification and olfactory sensitivity among NSHAP wave 2 olfactionsleep sub-sample $(n=345)$

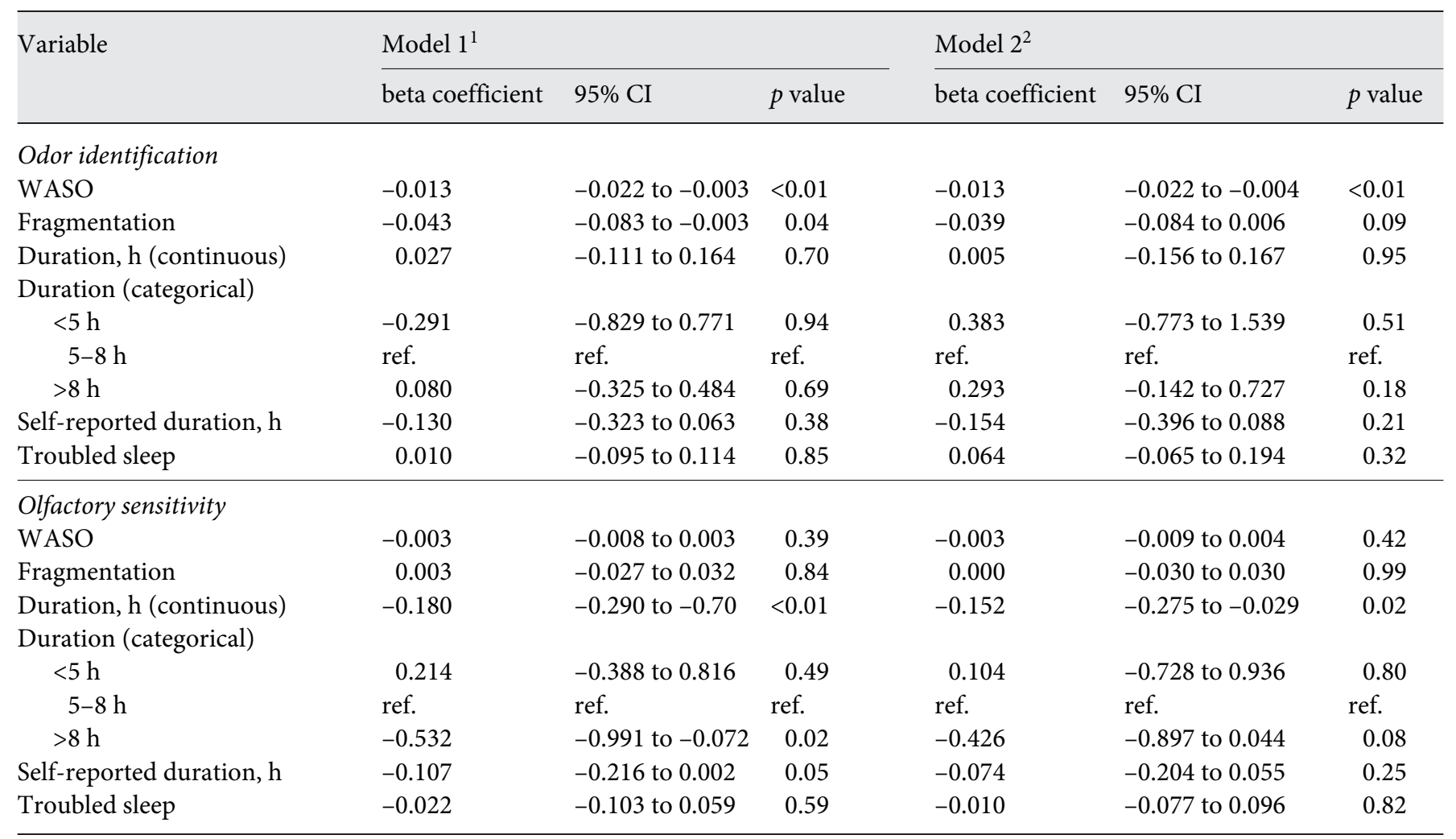

${ }^{1}$ Models are adjusted for age and gender.

${ }^{2}$ Models are further adjusted for race/ethnicity, BMI, a modified Charlson Comorbidity Index, Center for Epidemiologic Studies Depression Scale (CES-D), sleep medication and antidepressant usage, and current smoking status.

Table 3. Mediation of the associations between WASO and fragmentation and odor identification by cognition in NSHAP wave 2 olfaction-sleep subsample

\begin{tabular}{|c|c|c|c|}
\hline & $\begin{array}{l}\text { Beta } \\
\text { coefficient }\end{array}$ & $95 \% \mathrm{CI}$ & $p$ value \\
\hline A: Association with cognition & -0.034 & -0.056 , to 0.012 & $<0.01$ \\
\hline B: Association of cognition with odor ID (adjusting for WASO) & 0.063 & -0.006 to 0.131 & 0.07 \\
\hline Indirect association of WASO with odor ID through cognition & -0.002 & -0.005 to 0.001 & 0.14 \\
\hline \multicolumn{4}{|l|}{ Fragmentation } \\
\hline A: Association with cognition & -0.083 & -0.152 to -0.015 & 0.02 \\
\hline $\begin{array}{l}\text { B: Association of cognition with odor ID } \\
\text { (adjusting for fragmentation) }\end{array}$ & 0.073 & 0.007 to 0.140 & 0.03 \\
\hline $\begin{array}{l}\text { Indirect association of fragmentation with odor ID } \\
\text { through cognition }\end{array}$ & -0.006 & -0.012 to 0.003 & 0.22 \\
\hline
\end{tabular}


When considered a categorical variable, we found that the relationship was primarily driven by poorer odor sensitivity among long sleepers (8 or more hours per night). As a possible mechanism, we considered models controlling for reported neurological conditions (Parkinson's, Alzheimer's, and stroke) but found no difference in the estimated association. We also considered day-time napping behavior but found that this did not confound the observed associations. While the mechanism of the relationship between longer actigraph-measured sleep time and odor sensitivity remains unclear, there have been many reports of worse health outcomes among long sleepers [52].

We believe that this is the first study to assess the relationship between sleep and olfaction in a population of community-dwelling older adults and the first to include objective measures of both. Actigraph estimates of sleep characteristics avoid potential biases of survey sleep questions and are practical to implement in the field [53]. Similarly, the OFFE includes a validated measure of odor identification [39] and a novel survey-modified measure of odor sensitivity $[40,54]$. Although only a minority of NSHAP participants received both modularized measures, we did find several significant, and therefore unobserved, associations.

Our study did have some serious limitations. First, the data are cross-sectional, preventing the observation of temporal associations between disrupted sleep, olfaction, and cognition. Second, actigraphy cannot measure dimensions of sleep that may be salient for odor identification or sensitivity, such as sleep architecture. Third, only 354 of 3,196 age-eligible wave 2 NSHAP participants have both actigraphy and olfaction data, and a larger sample may be needed to detect associations with some of the sleep measures. Finally, NSHAP participants were not asked specifically about sleep apnea, although they were given an opportunity to list additional medical conditions. Only one participant in this subsample reported sleep apnea. There is likely to be both unreported and undiagnosed sleep apnea in the sample population, and we were unable to assess the role of sleep apnea in these associations.

Given the prior evidence that poor sleep could be a risk factor for cognitive impairment $[24,25,55]$ and that odor identification requires both detection and recognition (a cognitive function) [56,57], we explored whether the observed relationship between poor sleep quality and worse odor identification was mediated by cognition. Unexpectedly, it was not. It may be that the MoCA-SA does not adequately capture the cognitive components relevant to this pathway. Factor analysis has demonstrated that while the full MoCA captures more variability in MCI than screeners such as the Mini-Mental State Exam [58], it may not accurately identify domain-specific areas of cognitive impairment [59]. While impaired olfaction has been associated with global cognition, it has also been associated specifically with perceptual speed and episodic memory [60]. Thus, it is possible that sleep disruption does diminish capacity to identify odors through a cognitive pathway that is not well measured by the MoCA-SA. However, sleep disruption may affect olfactory processing centers independently of cognition. This alternative would suggest that the difference between the relationship of sleep to odor identification and odor sensitivity is not related to cognition.

Our study is broadly consistent with animal models on the role of sleep in the consolidation of odor memory. Barnes and Wilson found that manipulation via restriction of slow wave sleep in rats impaired memory consolidation relative to odor recognition [61]. Fragmentation and WASO may reflect lack of slow wave sleep. There are experimental studies of sleep restriction: Prehn-Kristensen et al. [27] and Killgore and McBride [35] found that sleep deprivation caused reduced capacity to recognize odors by adults. Our findings differ in that we found an association for lack of sleep consolidation (not sleep duration) and odor identification. Also, we examined routine sleep variation in the population rather than experimentally manipulated sleep.

Further study is needed to understand whether poor sleep quality leads to olfactory decline or vice versa, or whether underlying physiologic processes explain both, inducing the observed correlation between the 2. Longitudinal data would help answer these questions. If sleep disruption is an early indicator of olfactory dysfunction, it will be important to consider whether modifications to sleep routines aimed at improving sleep quality could delay the onset of other aging-related health outcomes.

\section{Acknowledgment}

This work was supported by R01AG042164 from the National Institute of Aging and the Basic Behavioral and Social Sciences Research Opportunity Network (OppNet) at the National Institutes of Health. The core data collection in the National Social Life and Aging Project (NSHAP) was supported by R01 AG021487 and R01 AG033903 from the National Institute of Aging at the National Institutes of Health. The ancillary sleep data collection for NSHAP received additional support from Phillips Respironics and the Health and Retirement Survey. 


\section{References}

1 Doucet S, Soussignan R, Sagot P, Schaal B: The secretion of Areolar (Montgomery's) glands from lactating women elicits selective, unconditional responses in neonates. PLoS One 2009; 4:e7579.

2 Jacob S, Garcia S, Hayreh D, McClintock MK: Psychological effects of musky compounds: comparison of androstadienone with androstenol and muscone. Horm Behav 2002;42: 274-283.

3 Patel RM, Pinto JM: Olfaction: anatomy, physiology, and disease. Clini Anat 2014;27: 54-60.

4 Kim WY, Hur M, Cho MS, Lee HS: Effect of olfactory function on nutritional status of Korean elderly women. Nutr Res 2003;23:723734.

5 Toussaint N, de Roon M, van Campen JP, Kremer S, Boesveldt S: Loss of olfactory function and nutritional status in vital older adults and geriatric patients. Chem Senses 2015;40: 197-203.

6 Boesveldt S, Lindau ST, McClintock MK, Hummel T, Lundström JN: Gustatory and olfactory dysfunction in older adults: a national probability study. Rhinology 2011;49:324330.

7 Sivam A, Wroblewski KE, Alkorta-Aranburu G, Barnes LL, Wilson RS, Bennett DA, Pinto JM: Olfactory dysfunction in older adults is associated with feelings of depression and loneliness. Chem Senses 2016;41:293-299.

8 Keller A, Malaspina D: Hidden consequences of olfactory dysfunction: a patient report series. BMC Ear Nose Throat Disord 2013;13:8.

9 Pinto JM, Wroblewski KE, Kern DW, Schumm LP, Mcclintock MK: The rate of agerelated olfactory decline among the general population of older U.S. adults. J Gerontol A Biol Sci Med Sci 2015;70:1435-1441.

10 Doty RL, Reyes PF, Gregor T: Presence of both odor identification and detection deficits in Alzheimer's disease. Brain Res Bull 1987; 18:597-600.

11 Attems J, Walker L, Jellinger KA: Olfaction and aging: a mini-review. Gerontology 2015; 61:485-490.

12 Wilson RS, Yu L, Bennett DA: Odor identification and mortality in old age. Chem Senses 2011;36:63-67.

13 Pinto JM, Schumm LP, Wroblewski KE, Kern DW, Mcclintock MK: Racial disparities in olfactory loss among older adults in the United States. J Gerontol A Biol Sci Med Sci 2014;69:323-329.

14 Devanand DP, Lee S, Manly J, Andrews H, Schupf N, Masurkar A, Stern Y, Mayeux R, Doty RL: Olfactory identification deficits and increased mortality in the community. Ann Neurol 2015;78:401-411.

15 Schubert CR, Fischer ME, Pinto AA, Klein BEK, Klein R, Tweed TS, Cruickshanks KJ: Sensory impairments and risk of mortality in older adults. J Gerontol A Biol Sci Med Sci 2017;72:710-715
16 Hoffman HJ, Rawal S, Li CM, Duffy VB: New chemosensory component in the U.S. National Health and Nutrition Examination Survey (NHANES): first-year results for measured olfactory dysfunction. Rev Endocr Metab Disord 2016;17:221-240.

17 Murphy C, Schubert CR, Cruickshanks KJ, Klein BE, Klein R, Nondahl DM: Prevalence of olfactory impairment in older adults. JAMA 2002;288:2307-2312.

18 Schubert CR, Cruickshanks KJ, Dalton DS, Klein BE, Klein R, Nondahl DM: Prevalence of sleep problems and quality of life in an older population. Sleep 2002;25:889893.

19 Floyd JA, Medler SM, Ager JW, Janisse JJ: Age-related changes in initiation and maintenance of sleep: a meta-analysis. Res Nurs Health 2000;23:106-117.

20 Ohayon MM, Carskadon MA, Guilleminault C, Vitiello MV: Meta-analysis of quantitative sleep parameters from childhood to old age in healthy individuals: developing normative sleep values across the human lifespan. Sleep 2004:27;255-273.

21 Kurina LM, McClintock MK, Chen JH, Waite LJ, Thisted RA, Lauderdale DS: Sleep duration and all-cause mortality: a critical review of measurement and associations. Ann Epidemiol 2013;23:361-370.

22 Rod NH, Vahtera J, Westerlund H, Kivimaki M, Zins M, Goldberg M, Lange T: Sleep disturbances and cause-specific mortality: results from the GAZEL cohort study. Am J Epidemiol 2011;173:300-309.

23 An C, Yu L, Wang L, Jin G, Song M, Zhu Q, Jia H, Liu K, Wang M, Wang X: Association between sleep characteristics and mild cognitive impairment in elderly people. Neurophysiology 2014;46:88-94.

24 Spira AP, Chen-Edinboro LP, Wu MN, Yaffe $\mathrm{K}$ : Impact of sleep on the risk of cognitive decline and dementia. Curr Opin Psychiatry 2014;27:478-483.

25 Blackwell T, Yaffe K, Ancoli-Israel S, Schneider JL, Cauley JA, Hillier TA, Fink HA, Stone $\mathrm{KL}$ : Poor sleep is associated with impaired cognitive function in older women: the study of osteoporotic fractures. J Gerontol A Biol Sci Med Sci 2006;61:405-410.

26 Knutson KL, Van Cauter E, Rathouz PJ, Yan LL, Hulley SB, Liu K, Lauderdale D: Association between sleep and blood pressure in midlife: the CARDIA Sleep Study. Arch Intern Med 2009;169:1055-1061.

27 Prehn-Kristensen A, Lotzkat K, Bauhofer E, Wiesner CD, Baving L: Sleep supports memory of odors in adults but not in children. PLoS One 2015;10:1-14.

28 Barnes DC, Wilson DA: Slow-wave sleep-imposed replay modulates both strength and precision of memory. J Neurosci 2014;34: 5134-5142.

29 Badia P, Wesensten N, Lammers W, Culpepper J, Harsh J: Responsiveness to olfactory stimuli presented in sleep. Physiol Behav 1990;48:89-90.

30 Stuck BA, Stieber K, Frey S, Freiburg C, Hörmann K, Maurer JT, Hummel T: Arousal responses to olfactory or trigeminal stimulation during sleep. Sleep 2007;30:506-510.

31 Rasch B, Sutton J: About sleep's role in memory. Psychologist 2014;27:320-323.

32 Rasch B, Büchel C, Gais S, Born J: Odor cues during slow-wave sleep prompt declarative memory consolidation. Science 2007;315: 1426-1429.

33 Diekelmann S, Biggel S, Rasch B, Born J: Offline consolidation of memory varies with time in slow wave sleep and can be accelerated by cuing memory reactivations. Neurobiol Learn Mem 2012:98;103-111.

34 Diekelmann S, Büchel C, Born J, Rasch B: Labile or stable: opposing consequences for memory when reactivated during waking and sleep. Nat Neurosci 2011;17:74-76.

35 Killgore WD, McBride SA: Odor identification accuracy declines following $24 \mathrm{~h}$ of sleep deprivation. J Sleep Res 2006;15:111-116.

36 Thomas ML, Sing HC, Belenky G, Holcomb $\mathrm{HH}$, Mayberg HS, Dannals RF, Wagner HN, Thorne DR, Popp KA, Rowland LM, Welsh AB, Balwinski SM, Redmond DP: Neural basis of alertness and cognitive performance impairments during sleepiness II: effects of 48 and $72 \mathrm{~h}$ of sleep deprivation on waking human regional brain activity. Thalamus Relat Syst 2003;2:199-229.

37 O’Doherty K, Jaszczak A, Hoffmann JN, You HM, Kern DW, Pagel K, McPhillips J, Schumm LP, Dale W, Huang ES, Mcclintock MK: Survey field methods for expanded biospecimen and biomeasure collection in NSHAP Wave 2. J Gerontol B Phychol Sci Soc Sci 2014;69:S27-S37.

38 Kern DW, Wroblewski KE, Schumm LP, Pinto JM, Mcclintock MK: Field survey measures of olfaction: the Olfactory Function Field Exam (OFFE). Field methods 2014;26:421434.

39 Mueller C, Renner B: A new procedure for the short screening of olfactory function using five items from the "Sniffin' Sticks" identification test kit. Am J Rhinol 2006;20:113-116.

40 Kern DW, Schumm LP, Wroblewski KE, Pinto JM, Hummel T, McClintock MK: Olfactory thresholds of the U.S. population of homedwelling older adults: development and validation of a short, reliable measure. PLoS One 2015; 10:e0118589.

41 Pinto JM, Kern DW, Wroblewski KE, Chen RC, Schumm LP, Mcclintock MK: Sensory function: insights from wave 2 of the National Social Life, Health, and Aging Project. J Gerontol B Psychol Sci Soc Sci 2014;69:144-153.

42 Lauderdale DS, Schumm LP, Kurina LM, McClintock M, Thisted RA, Chen JH, Waite L: Assessment of sleep in the National Social Life, Health, and Aging Project. J Gerontol B Phychol Sci Soc Sci 2014;69(suppl 2):S125-S133. 
43 Chen JH, Waite L, Kurina LM, Thisted RA, Mcclintock MK, Lauderdale D: Insomnia symptoms and actigraph-estimated sleep characteristics in a nationally representative sample of older adults. J Gerontol A Biol Sci Med Sci 2015;70:185-192.

44 Nasreddine ZS, Phillips NA, Bedirian V, Charbonneau S, Whitehead V, Collin I, Cummings JL, Chertkow H: The Montreal Cognitive Assessment, MoCA: a brief screening tool for mild cognitive impairment. J Am Geriatr Soc 2005;53:695-659.

45 Shega JW, Sunkara PD, Kotwal A, Kern DW, Henning SL, Mcclintock MK, Schumm P, Waite LJ, Dale W: Measuring cognition: the Chicago cognitive function measure in the national social life, health and aging project, wave 2. J Gerontol B Psychol Sci Soc Sci 2014; 69(suppl 2)S166-S176.

46 Kotwal AA, Schumm P, Kern DW, Mcclintock MK, Waite LJ, Shega JW, Huisingh-Scheetz MJ, Dale W: Evaluation of a brief survey instrument for assessing subtle differences in cognitive function among older adults. Alzheimer Dis Assoc Disord 201529:317-324.

47 Charlson ME, Pompei P, Ales KL, MacKenzie CR: A new method of classifying prognostic comorbidity in longitudinal studies: development and validation. J Chronic Dis 1987;40: 373-383.

48 Vasilopoulos T, Kotwal AA, HuisinghScheetz MJ, Waite LJ, Mcclintock MK, Dale $\mathrm{W}$ : Comorbidity and chronic conditions in the national social life, health and aging project (NSHAP), wave 2. J Gerontol B Psychol Sci Soc Sci 2014;69(suppl 2):S154-S165.

49 Cappuccio FP, D’Elia L, Strazzullo P, Miller MA: Sleep duration and all-cause mortality: a systematic review and meta-analysis of prospective studies. Sleep 2010;33:585-592.

50 Baron RM, Kenny DA: The moderator-mediator variable distinction in social psychological research: conceptual, strategic, and statistical considerations. J Pers Soc Psychol 1986; 51:1173-1182.

51 O'Muircheartaigh C, English N, Pedlow S, Kwok PK: Sample design, sample augmentation, and estimation for Wave 2 of the NSHAP. J Gerontol B Psychol Sci Soc Sci 2014; 69(suppl 2):S15-S26.

52 Ramos AR, Tarraf W, Daviglus M, Davis S, Gallo LC, Mossavar-Rahmani Y, Penedo FJ, Redline S, Rundek T, Sacco RL, Sotres-Alvarez D, Wright CB, Zee PC, Gonzalez HM: Sleep duration and neurocognitive function in the Hispanic Community Health Study/ Study of Latinos. Sleep 2016;39:1843-1851.

53 Ancoli-Israel S, Cole R, Alessi C, Chambers M, Moorcroft W, Pollak CP: The role of actigraphy in the study of sleep and circadian rhythms. Sleep 2003;26:342-392.

54 Kern DW, Wroblewski KE, Schumm LP, Pinto JM, Chen RC, McClintock MK: Olfactory function in wave 2 of the national social life, health, and aging project. J Gerontol B Psychol Sci Soc Sci 2014;69(suppl 2):S134-S143.
55 Blackwell T, Yaffe K, Ancoli-Israel S, Redline S, Ensrud KE, Stefanick ML, Laffan A, Stone KL: Association of sleep characteristics and cognition in older community-dwelling men: the MrOS sleep study. Sleep 2011;34:13471356.

56 Devanand DP, Tabert MH, Cuasay K, Manly JJ, Schupf N, Brickman AM, Andrews $\mathrm{H}$, Brown TR, DeCarli C, Mayeux R: Olfactory identification deficits and MCI in a multi-ethnic elderly community sample. Neurobiol Aging 2010;31:1593-1600.

57 Swan GE, Carmelli D: Impaired olfaction predicts cognitive decline in nondemented older adults. Neuroepidemiology 2002;21: 58-67.

58 Folstein MF, Folstein SE, McHugh PR: "Minimental state". A practical method for grading the cognitive state of patients for the clinician. J Psychiatr Res 1975;12:189-198.

59 Coen RF, Robertson DA, Kenny RA, KingKallimanis BL: Strengths and limitations of the MoCA for assessing cognitive functioning: findings from a large representative sample of Irish older adults. J Geriatr Psychiatry Neur 2016;29:18-24.

60 Wilson RS, Arnold SE, Tang Y, Bennett DA: Odor identification and decline in different cognitive domains in old age. Neuroepidemiology 2006;26:61-67.

61 Barnes DC, Wilson DA: Sleep and olfactory cortical plasticity. Front Behav Neurosci $2015 ; 8: 134$ 